\title{
A Novel Spectrum Decision Pattern for The Favorable Cognitive Radio Based Internet of Things in 5G System
}

\author{
Hyils Sharon Magdalene. A, D. Shalini Punithavathani
}

\begin{abstract}
The Radio Frequency (RF) Spectrum decision in Cognitive Radio (CR) allows unlicensed users of wireless communication schemes to conquer the unoccupied spectrum slots as a resolution for barely spectrum. The Internet of Things (IoT) is a broad-arriving network and its object is linked by wireless communication technologies, donating cost- efficiency and generously opens to remote users. When IoT is applied, it is injured by challenges of susceptibility in the forceful surrounding situations, allotment and use of bandwidth, ease of approach and expense to buy $R F$ spectrum. The object is permeated with cognitive capacity and is capable to model RF spectrum decisions to attain interference-release and wireless connectivity due to their Quality of Service (QoS) demands. Therefore the spectrum decision through an unlicensed user on CR influences an importance in CR-based IoT in $5 G$ system and further network. This article depicts a systematic sustainment of the spectrum decision structure to CR Network. So, this can be mainly achieved by applying a favorable spectrum sensing technique and a novel spectrum decision framework. Presently, the wireless connectivity is intended to greater capacity, immense machine connectivity, and greater data range, low end-to-end latency, low cost and coherent Quality of Experience (QoE) condition. Hence, $4 G$ is being substituted by 5G. Being in this 5G, the vast connectivity can be properly inspired by using the novel techniques, which is applied under the Energy detection spectrum sensing system. Also, a novel spectrum decision framework is designed for the optimum application of applying the IoT in $5 G$ system ie., the effective use of an allocated $R F$ spectrum is differently underutilized because of the standard handling with the licensed users are called as Primary Users (PUs).

Keywords: Cognitive Radio, Internet of Things, IoT-User, 5G/B5G.
\end{abstract}

\section{INTRODUCTION}

The 4G supplies an information, voice and a multimedia which is given to the wireless subscribers toward all period and in every place founded at greater data ranges in multimedia intimation service, video conversation, Digital Video conversation Broadcasting (DVB), mobile television (mobile TV) and for superior limited TV. In the usage of 5G network, the wireless schemes are spread out to model every humans and objects to be joined at every time to everyone while existing everywhere via every route or network and

Manuscript received on June 11, 2021

Revised Manuscript received on June 17, 2021.

Manuscript published on June 30, 2021.

* Correspondence Author

Hyils Sharon Magdalene. A*, Department of Computer Science \& Engineering, PSN College of Engineering and Technology, Melathediyoor (Tamil Nadu), India. Email: hyils12@gmail.com

Dr. D. Shalini Punithavathani, Department of Electronics \& Communication Engineering, Government College of Engineering, Tirunelveli (Tamil Nadu), India. Email: shalini329@gmail.com

(C) The Authors. Published by Blue Eyes Intelligence Engineering and Sciences Publication (BEIESP). This is an open access article under the CC BY-NC-ND license (http://creativecommons.org/licenses/by-nc-nd/4.0/)

every services including A6 junction. So, this A6 junction is called as IoT. The IoT has the surrounding in which an every above smart interconnected thing is joined to everyone by exceptional addressing systems that depends on definite telecommunication basis and protocols [1]. The IoT depended objects are to be interlinked by Base Transceiver Stations (BTSs) in wireless functions and the BTSs are connected to backhaul linking by Optical Fiber communication schemes which gains greater bandwidths added by Terrestrial Microwave connectivity. The wireless Radio Frequency spectrum (WRFS) is entirely allocated to existing wireless usages. Simultaneously, the WRFS is underutilized because of the classic use of mobile and another wireless functions. To overcome the difficulty, the CR is appeared as a possible technology that suggests a resolution for the troublesome of spectrum scarcity.

The Cognitive Radio Network (CRN) systems are revealed as a proficient solution for the spectrum scarcity and as a possible technology for the best usage of differentially underutilized RF spectrum, humanizing the interoperability and synchronicity in different mobile and wireless transmission methods converting towards long-distance communication machines, self-reconfigurable and systems independency. The Secondary Users (SUs) approach RF spectrum bands in various modes of CRN and IoT aided smart regions composed of different machines that are fixed as well as portable in nature [1]. The 5G and further network is assumed to incorporate the modern wireless technologies towards an every Internet Protocol (IP) established networks which gives greater operating worldwide network [2]. Simultaneously, the future generation mobile transmission network is mentioned to as the Fifth Generation (5G) which is nearly understood in the modern telecommunication period of time [3]. The bandwidth of 5G and further network is extremely great and the WRFS gives a large number of non-uninterrupted vacant spectrum slots in 5G transmission as well, there is a demand to recognize the unoccupied spectrum slots not existing utilized by corresponding licensed users known as PUs [4].

This method is called as Spectrum Sensing (SS) in CR schemes. The precise SS enables the SUs to occasionally apply an unoccupied spectrum slots due to their wireless functions and leave when the Primary User (PU) reaches in the network.

Blue Eyes Intelligence Engineering and Sciences Publication

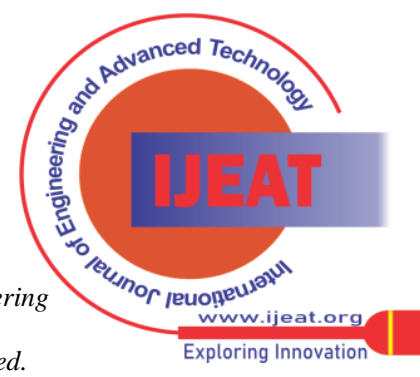


This method is referred to as spectrum decision. When possibly acted, the SU together with the PU will allow users by applying IoT model in 5G/ B5G (Beyond $5^{\text {th }}$ Generation) networks. Hence, the spectrum decision is a significant variable for the strategic positioning of CR-depended IoT in 5G/B5G network. The RF spectrum accessibility due to the wireless usage for the user in IoT surrounding remains an available experimental domain. An extensive survey of 5G networks initialized with IoT usages established on CR assuring A6 linking through accessing beyond the whole RF spectrum has been carried in this article.

In addition, a practical cooperative spectrum sensing network is designed for the optimum spectrum decision model where the reporting channels from the cognitive radios (CRs) to the Cognitive Base Station (CBS) are infected through the Rayleigh fading and Additive White Gaussian Noise (AWGN). The spectrum decision model is a significant thing for the embedded application of the CR-IoT in 5G/B5G network. In this paper, the optimum spectrum decision model is carried out for the better operation of CR-depended IoT in 5G/B5G network.

\section{ORGANIZATION OF AN ARTICLE}

The first part of an article provides an introduction, associated work and the task motivation. The third part delivers the development toward 5G system and the fourth part depicts the IoT to $5 \mathrm{G}$ network. The fifth part delivers an agreement to $5 \mathrm{G}$ system having CRN founded IoT and the sixth part provides a necessity of RF spectrum operation. The seventh part considers the optimum spectrum decision framework for the enhancement of the system. Then, the article is concluded in eight part.

\section{WIRELESS TECHNOLOGIES IN DEVELOPMENT SINCE 1G - 5G}

In the beginning of the first generation (1G) cellular systems in telecommunication scheme, the whole design of existing surroundings involves the human's task, agriculture, lifestyle and an industrial evolution mode has been executed. The growth of the fifth generation (5G) is the advancement in the telecommunication industry to possess with the growing step on enormous amount of machine junctions, mobile data traffic and uninterrupted appearance of recent business scenarios. At the end of the last decade and so, the wireless communications have the ability to join every existing mobile technologies, to construct a terminal that is to help the video, voice and data usage that having corresponding QoS needs and is assured i.e., at extremely large data ranges and users speeds doing this as 5G/B5G environment. The Table I inscribed a chronological development of 5G/B5G environment [5]. An appearance of 5G wireless communication visualizes enormous data range, having the characteristics of Gbps group, very low latency, considerable growth in BTS volume and an enhancement in PUs', SUs' felt QoE, which is similar to being 4G/3G wireless networks. The 5G/B5G suggests the total wireless world mutual connection as Wireless Innovative System for Dynamic Operation Mega communications (WISDOM) concept, having assured QoS demands of wireless functions [6]. The spectrum decision in CR assures the spectrum scarcity troublesome and IoT consent wireless A6 link for users and making CR-established IoT in 5G networks that having an adjust on spectrum decision model in CR to IoT system in $5 G$ networks.

\section{IOT SYSTEM INTO 5G/B5G NETWORKS}

An IoT and a mobile data are the internet for the coming event of everything and stimulating a power in the progress of 5G/B5G networks. In the near future, probably from 2021 to 2025 , 5G/ B5G network not even encounter the classified demands of humans in several constituencies of everyday life as work, residence, recreation and conveyance, but too will pour the IoT and illuminated the different proficient field in the professional appearances of people life and the industry such as medical sciences and resources and conveyance to understand the exact interconnectedness of every matters [7]. The fulfillments of IoT is relying on an internet usage scenario that establishes demands to meet $5 G$ networks and are not assured in LTE and 4G technologies. These demands are inscribed in Table II [8].

Table-II: Advanced mode and demands in IoT

\begin{tabular}{|c|c|c|c|c|}
\hline \multirow[b]{2}{*}{$\begin{array}{l}\text { Internet } \\
\text { usage } \\
\text { circumst } \\
\text { ances }\end{array}$} & \multicolumn{2}{|c|}{$\begin{array}{c}\text { Mobile data supplied } \\
\text { internet to the } \\
\text { subscribers }\end{array}$} & \multicolumn{2}{|c|}{ IoT } \\
\hline & $\begin{array}{l}\text { Broad and } \\
\text { seamless } \\
\text { coverage }\end{array}$ & $\begin{array}{c}\text { Increasin } \\
\text { g capacity } \\
\text { to ensure } \\
\text { QoS } \\
\text { needs for } \\
\text { Internet } \\
\text { Usages } \\
\end{array}$ & $\begin{array}{c}\text { A6 } \\
\text { junction }\end{array}$ & $\begin{array}{l}\text { Less end } \\
\text { to end } \\
\text { latency }\end{array}$ \\
\hline Demands & $\begin{array}{l}\text { Seamless } \\
\text { linking with } \\
\text { greater } \\
\text { speed } \\
\text { function in } \\
\text { mobility of } \\
\text { the } \\
\text { subscriber }\end{array}$ & $\begin{array}{l}\text { Massively } \\
\text { greater } \\
\text { data } \\
\text { communi } \\
\text { cation } \\
\text { range }\end{array}$ & $\begin{array}{l}\text { Providing } \\
\text { linking to } \\
\text { billions of } \\
\text { machines } \\
\text { with } \\
\text { suitable } \\
\text { capacity } \\
\text { of power } \\
\text { needs for } \\
\text { hardware } \\
\text { compone } \\
\text { nts }\end{array}$ & $\begin{array}{l}\text { Providing } \\
\text { service to } \\
\text { users with } \\
\text { lower than } \\
\text { millisecon } \\
\text { d } \\
\text { end-to-end } \\
\text { delays in } \\
\text { communic } \\
\text { ation and } \\
\text { in } \\
\text { swapping } \\
\text { of } \\
\text { spectrum } \\
\text { slots }\end{array}$ \\
\hline
\end{tabular}

\section{5G/B5G IN CRN DEPENDED IOT}

The IoT visualizes thousands of limited devices with motivating, sensing, adapting, and transferring abilities which is capable of monitoring the globe with a novel decision.

Correspondingly to Cisco, greater than 50 billion machines are anticipated to be joined to the internet on 2020 and $20 \%$ of whatever by the industrial region [7]. These joined objects will produce enormous amount of data that is necessarily to be examined to achieve perceptive behind this large IoT information.

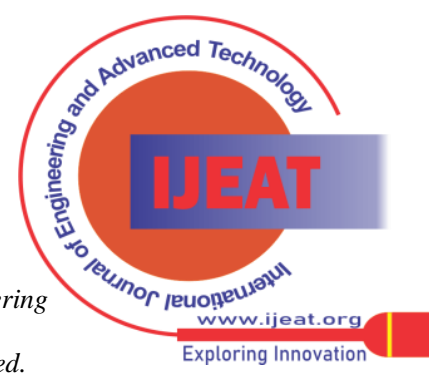


In addition, smart spaces (construction, building, etc.) as well as in the industrial surroundings (industry 4.0) and linked car transmissions frequently need less latency, high dependability and scalability. Various technologies like Zigbee, IPv6 settings integrated with Time Synchronized Channel Hopping (6TiSCH), Bluetooth Low Energy (BLE), Low-Power Wide Area Network (LPWAN), Long Range (LoRa), etc., Wireless Highway Addressable Remote Transducer (HART) Protocol, are suggested to adapt these necessities. The available $5 \mathrm{G}$ networks are assured not only in enlarging data ranges but also having less latency data transmission for latency-crucial IoT usages.

Table-I: Advanced development of Mobile operation from 1G to 5G [5]

\begin{tabular}{|c|c|c|c|c|c|}
\hline technology & Usages & Criterion & $\begin{array}{l}\text { Data } \\
\text { ranges }\end{array}$ & $\begin{array}{l}\text { Mobility } \\
\text { suggested }\end{array}$ & $\begin{array}{l}\text { Time } \\
\text { duration }\end{array}$ \\
\hline 1G (Analog) & $\begin{array}{l}\text { 1st generation of the } \\
\text { mobile telecommunication } \\
\text { technology normalized by } \\
\text { the voice service }\end{array}$ & 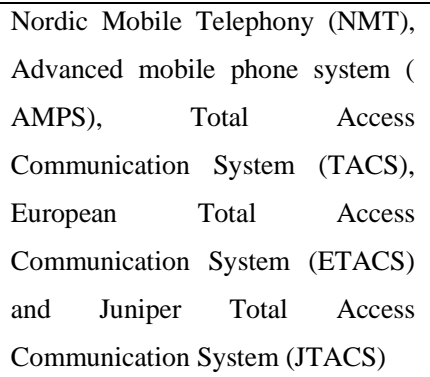 & $14.4 \mathrm{kbps}$ & Less speed & 1995 - 1997 \\
\hline 2G (Digital) & $\begin{array}{l}\text { 2nd Generation of wireless } \\
\text { telephone } \quad \text { technology } \\
\text { initiating a } r \text { detailed } \\
\text { service; SMS } \\
\text { message service) }\end{array}$ & $\begin{array}{l}\text { Time Division Multiple Access } \\
\text { (TDMA), Global System for Mobile } \\
\text { Communications (GSM), Code } \\
\text { Division Multiple Access (CDMA), } \\
2.4 \text { GHz narrow band Wireless } \\
\text { Local Area Network (WLAN) }\end{array}$ & $144 \mathrm{kbps}$ & $\begin{array}{l}\text { Less and } \\
\text { medium speed }\end{array}$ & $1997-2000$ \\
\hline $\begin{array}{l}\text { 3G - IMT } 2000 \text { (International } \\
\text { mobile Telecommunications - } \\
\text { 2000) }\end{array}$ & $\begin{array}{l}\text { 3rd generation of mobile } \\
\text { telecommunication (IMT - } \\
\text { 2000) }\end{array}$ & $\begin{array}{l}\text { CDMA 2000, Evolution Data } \\
\text { Only/Evolution Data Optimized } \\
\text { (EV-DO), Wideband Code Division } \\
\text { Multiple Access (W-CDMA), } \\
802.11 \quad \text { personal area network } \\
\text { (PAN), Bluetooth. }\end{array}$ & 384 kbps & $\begin{array}{l}\text { Medium and } \\
\text { increasing } \\
\text { speed }\end{array}$ & $2000-2005$ \\
\hline B3G & Beyond 3rd generation & 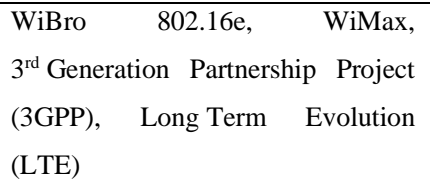 & $\begin{array}{l}<50 \\
\text { mbps }\end{array}$ & Greater speed & $2005-2010$ \\
\hline $4 \mathrm{G}$ & $\begin{array}{l}\text { 4th generation of mobile } \\
\text { telecommunications }\end{array}$ & $\begin{array}{l}\text { Digital audio broadcasting (DAB), } \\
\text { Digital Video Broadcasting (DVB), } \\
\text { cellular GSM, IMT - 2000, WLAN, } \\
\text { Infrared (IR), ultra wide band } \\
\text { (UWB), Digital Subscriber Line } \\
\text { (DSL), LTE-A, IEEE 802.16e }\end{array}$ & $\begin{array}{l}<100 \\
\text { mbps }\end{array}$ & $\begin{array}{l}\text { Very } \\
\text { speed }\end{array}$ & $\begin{array}{l}2010 \\
\text { onwards }\end{array}$ \\
\hline B5G & 5th generation and further & $4 \mathrm{G}+$ WISDOM & & & $\begin{array}{l}2015 \\
\text { onwards }\end{array}$ \\
\hline
\end{tabular}

The 5G will allow massive IoT devices linked via a many of networks and crucial machine sort transmissions. If the massive IoT is greatly interested about scalability far covering and energy efficiency, the former demands an ultra-low latency and much credibility (URLLC). Lately, the fog-to-object sequence is suggested [9] to reduce the weighty load on the network because of the centralized adapting and accumulating the enormous IoT information. The fog-allowed IoT structures ensures near processing in closeness of the objects, which have the solutions of small, deterministic retrieval time that allows real time usages and strengthen security.

Published By:

Blue Eyes Intelligence Engineering and Sciences Publication

(C) Copyright: All rights reserved.

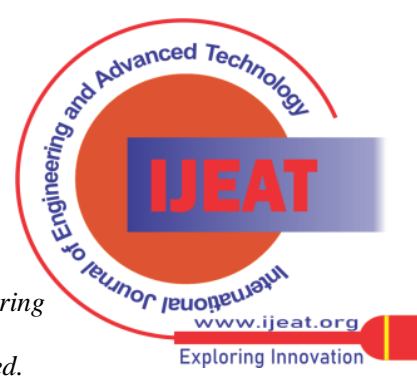


Due to the principal sight of the IoT, the advanced need is the ubiquity of the Internet, behind linking humans at every time and in every place, to link nonexistent entities.

The mission of spectrum for a large number of machines and objects needed for IoT connectivity will effects in additional cost impact. The CRNs owned to its standard spectrum usage attribute appear in fulfillment of IoT. The conception of a conserved spectrum slot as Reserved-to-Share (RS) and Shared-to-Reserve (SR) systems in Cognitive Radio-Heterogeneous Networks (CR-HetNets) suggested in Habibzadeh et al. (2017) can improves the system rate of transfer and would give a greater bandwidth communication to IoT-Users (IoT-Us) in CRN. A penetrating and distributed network of faultlessly linked varied electronic and electric devices is formed, in what is to be naturally adhesive toward the existent mobile networks and Internet junctions. Conventionally, IoT can be explained as, "A universal network on electronically linked machines exceptionally addressable that depends on typically transferred protocols and enables users to be A6 linked" [11]. Recent research task and technological methods are meeting to IoT and CRNs.

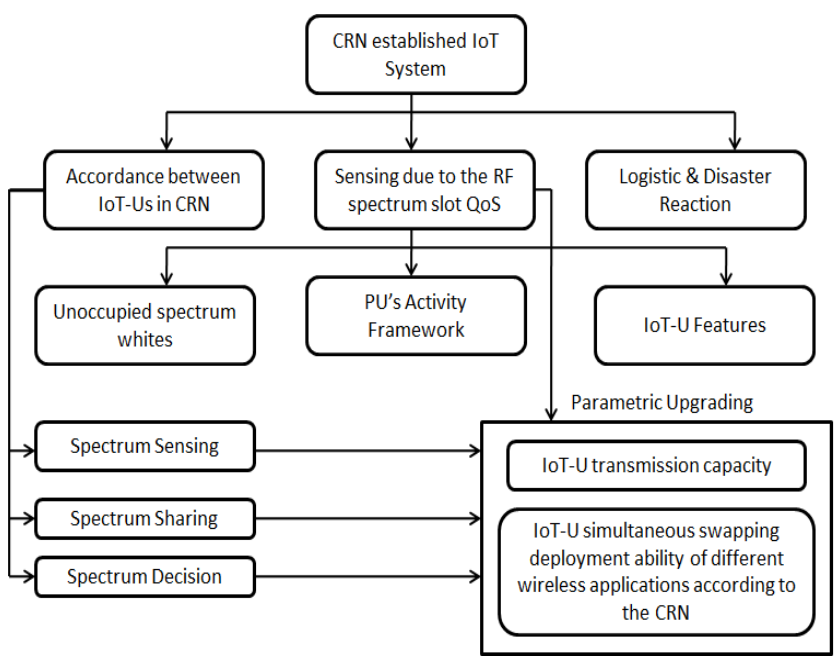

Fig. 1.CRN system among its features and experimental guidance allowing it for IoT system in A6 linking through SUs.

A CRN characteristic allowing it for IoT usages are represented in Figure 1. Generally, the SU performs in Half-Duplex (HD) mode, i.e., it can either communicate or sense on every moment of period [12]. Because of this HD performance on SU, there is a probability that damaging intervention to PU is happened on sudden reach of PU in the system and its activity throughout the communication of SU. Therefore the spectrum sensing must be an uninterrupted process and SU should leave the licensed channel while an appearance of its PU and swap to other compatible channel due to its usage, i.e., a proper spectrum decision model is needed.

\section{REQUIREMENT FOR SPECTRUM MANAGEMENT IN IOT DEPEND 5G/B5G SYSTEMS}

The exterior storage resolutions gives almost limitless capability, having devoted signal processing to group by finding signals and data, communications or occurrences of interest. This extended period with greater bandwidth solutions are prototype for modern technologies like cognitive radios and current crowded spectrum. Conserving the demanded QoS of the users together with their mobility claims spectrum mobility for the SUs in the network, in what is currently called as 5G network. Due to its mobility, the SU may alter its position (cell) in a cellular network while its communication and, hence, will go into a modern field in whichever the aimed RF spectrum slot is previously utilized by the PU [13].

The WRFS is described through exhibit of PU activity and precise SS [14]. This intends that the spectrum management includes a huge importance in A6 junction and CR technologies. The Dynamic Spectrum Access (DSA) enables both the SUs and PUs to possibly utilize the spectrum slots while assuring their QoS needs. The flawless SS techniques supplies previous data in order to what SUs will act [5]. The SUs contains the real time data of RF spectrum slots of possession status to swap over to the unoccupied slots for carry on their communication in case PUs reaches.

Correspondingly, the SS defects are needed to be restricted. The determination of accessing the unoccupied spectrum slots allows the SU with A6 connections to make IoT surroundings. Hence, the $\mathrm{SU}$ is renamed behold as IoT-User (IoT-U). The formal access to spectrum management is extremely rigid in the sense in which every wireless service provider allotted a solitary license to perform in a particular frequency spectrum band [15]. By applying CR systems, the method of spectrum usage demands a Dynamic Spectrum Management Framework (DSMF) that contains four major constituents (identifying Spectrum Decision structure, SS, spectrum mobility and spectrum sharing) [16]. Hence, for effective use of the spectrum making opportunities, Cognitive Radio (CR) technology enables its users known as SUs to conquer the unoccupied spectrum slot for its transmission in order to the framework is exhibited in Figure 2 and depart it on appearance of the licensed user (known as PU) [17].

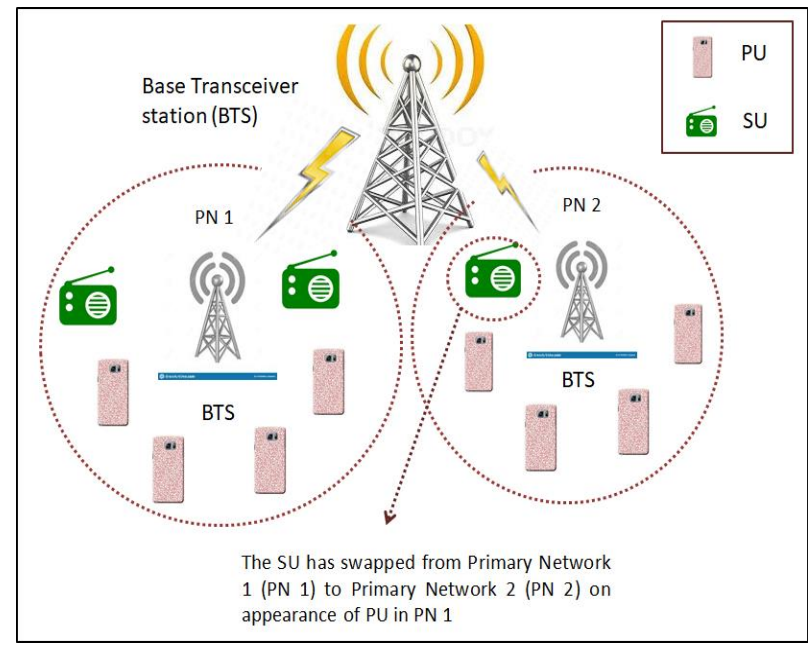

Fig. 2.Processing of a CRN framework in which the $\mathrm{SU}$ is swapped to other network on reaching of PU in Primary Network 1(PN 1)
Published By:

Blue Eyes Intelligence Engineering and Sciences Publication

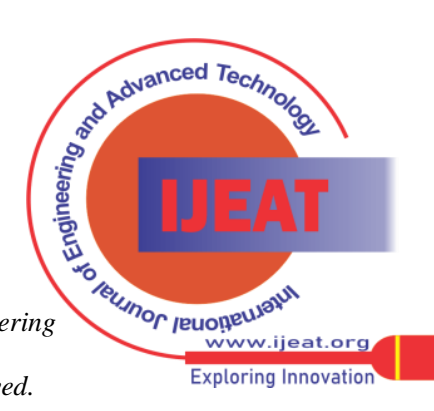


The spectrum mobility allows the SU to swap over to another channel whereas a PU is reached. The spectrum sharing is mentioned as a coordinated approach towards the chosen channel by the SUs. The SS includes recognition of spectrum holes and the capability to rapidly find the beginning of PU transmissions in the channels conquered by the SUs. The spectrum decision allows SUs in CRN to choose the optimum unoccupied spectrum slots to fulfill SUs' QoS demands without happening damaging interference to the PUs [18]. The SU holds its communication during the PU semblance in the spectrum slot. The SU observe (which demands an effective SS) and choose (which claims an appropriate Spectrum Decision structure) additional QoS consent to spectrum slot whatever is occupied/not. This suggests that there are two stages (spectrum decision structure and SS) of effective use of spectrum in CR-depended IoT of 5G/B5G network.

There are two types of scenarios for spectrum sharing such as underlay and overlay spectrum sharing. In one scenario the SU exists together with the PU and communicates with power limitations to assured the QoS of the PU [19]. This is called as underlay spectrum sharing. The overlay way of performance is centered in this paper. In other scenarios, the SU accesses a RF spectrum slot solely when it is not exist utilized by the PU [20]. This is occurred in the spectrum overlay scenarios. This scenario is also called as opportunistic spectrum access.

\section{A. Spectrum Sensing}

The CR strategy suggests the ability of IoT-Us to enhance the spectrum usages according to the previous static spectrum allocation policy. The IoT-Us not only sense the spectrum surroundings nearby and access unoccupied spectrum slots in the favorable way, however also need to sense the appearance of PU's signal sequentially to possess the SS data upgraded. Henceforth, the SS is the basic demand in CR and the establishment to Spectrum Decision. The SS methods are classified into cooperative (non-centralized detection and Centralized Spectrum Sensing (CSS)), interference detection and non-cooperative (Matched Filter Detection (MFD), Energy Detection (ED) and cyclostationary detection) [21].

The enhanced ED and CRs with several antennas will expand the SS execution [22]. The ED and MFD operated on consistent (by estimating the energy of the received signal instances) and non-consistent (comparing with the recognized PU signal) detection correspondingly [23]. The non-centralized detection and CSS have proved the SS flaws because of the time delay that included among sensing and its outcomes [24]. In this article, the novel scheme of SS is designed under the ED for the better outcome of spectrum decision framework.

The cyclostationary detection inflicts from larger complexity as an every cycle frequencies are need to be estimated [25]. Although the unoccupied spectrum slots are recognized in SS if before these are not concurrently conquered by a well-specified decision method, the idea of $\mathrm{CR}$ is not able to be understood. Hence, it is commanded to lessen the SS errors (miss detection and false alarm) before holding the decision to conquer the sensed unoccupied spectrum slot.

\section{B. PUs and IoT-Us Activity Framework}

There are two kinds of users in CRN as PU and SU to utilize the WRFS, in what have been taken as IoT-U in this paper. Then, Federal Communications Commission (FCC) has agreed the entry of an unlicensed users (IoT-Us) to the previously sold RF spectrum, that supplied the unlicensed users which do not happen damaging interference to the PUs. The execution of the CRNs is greatly based on PU which reaches and leaves from the spectrum slots; the license of what it includes [26]. Therefore it's significant to make pattern for the PU activity of CRNs to allow IoT-Us to decide for the possession of idle spectrum slots. The PUs in the broader field of WRFS performed in any spectrum which is based on the particular wireless usages. The functioning of radio frequency bands in several wireless technologies is demonstrated in Table III.

Table-III: Frequency spectrum rates for different wireless implementations [8]

\begin{tabular}{|c|c|c|}
\hline $\begin{array}{c}\text { Wireless } \\
\text { Applications }\end{array}$ & $\begin{array}{c}\text { Frequency spectrum } \\
\text { bands }\end{array}$ & Bandwidth \\
\hline $\begin{array}{l}\text { IEEE 802.11g to } \\
\text { n/WiFi }\end{array}$ & $2.4 \mathrm{GHz}$ & $10 \mathrm{KHz}$ \\
\hline $\begin{array}{l}\text { IEEE } \\
802.16 / \mathrm{LAN} / 2\end{array}$ & $5 \mathrm{GH}$ & $100 \mathrm{KHz}$ \\
\hline IEEE 802.22 & 54-862 MHz & 5-20 MHz \\
\hline GSM & $\begin{array}{l}\text { 890-915 MHz (uplink) } \\
\text { 935-960 } \\
\text { (downlink) }\end{array}$ & $200 \mathrm{KHz}$ \\
\hline CDMA & 800 and $1.9 \mathrm{GHz}$ & $125 \mathrm{MHz}$ \\
\hline W-CDMA & $850-2100 \mathrm{MHz}$ & $\begin{array}{l}125 \mathrm{MHz}, 250 \\
\mathrm{MHz}\end{array}$ \\
\hline LTE & $\begin{array}{lll}1710- & 1770 & \mathrm{MHz} \\
\text { (uplink) } & & \\
2110-2170 & \mathrm{MHz} \\
\text { (downlink) } & & \\
\end{array}$ & $20 \mathrm{MHz}$ \\
\hline UWB & $3.1-10.6 \mathrm{GHz}$ & $500 \mathrm{MHz}$ \\
\hline 5G Cellular & $\begin{array}{l}26.5-40 \mathrm{GHz} \text { and } 30-50 \\
\mathrm{GHz}\end{array}$ & $\begin{array}{l}\text { Total rates of } \\
\text { bandwidths i.e., } \\
\text { wide, narrow, } \\
\text { super ultra wide } \\
\text { band and ultra } \\
\text { wide bands } \\
\text { transmission } \\
\text { systems }\end{array}$ \\
\hline
\end{tabular}

\section{SPECTRUM DECISION STRUCTURE}

The CR technology is described by SUs and PUs, an accordance between the IoT-Us to apply the licensed bands whereas the PUs are not utilizing the spectrum slots in the objective spectrum slot. Despite, in the latest periods, the investigators have continued work in the region of Spectrum Decision; anyway, but this region is not yet totally examined. A review of spectrum decision in CRNs depended on RF spectrum description, spectrum assortment and CR reformation has been demonstrated [18].

Since (2013), the origin of work is based on a fuzzy inference-founded decision strategy that depends on three fundamental variables as, spectrum slot occupancy position, spectrum slot QoS and spectrum slot unoccupancy period is one of the spectrum decision frameworks [27].

Published By:

Blue Eyes Intelligence Engineering and Sciences Publication

DOI:10.35940/ijeat.E2808.0610521

Journal Website: www.ijeat.org

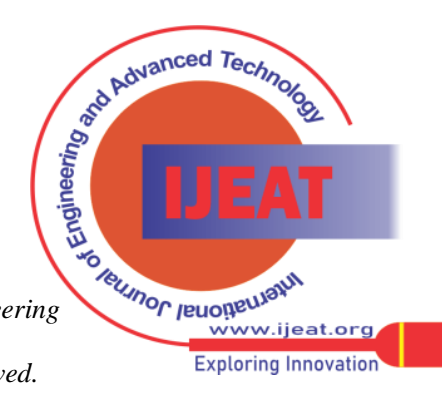


This supplies a precise and strong spectrum decision system for SUs.

An identical and can be equally efficient for IoT-Us in CR-depended IOT in 5G/B5G networks as it encloses every signal adapting matrices like wireless dispersion channel frameworks, differently bandwidths traits and Massive Multiple input multiple output (MIMO) Antennas for every wireless usages (by QoS spectrum slot), needed for wireless transmissions. In addition, a spectrum decision strategy is suggested here with an examination system. This system is depended on combination of three distinct decisions of three fundamental variables as, spectrum slot occupancy position, spectrum slot execution and the spectrum slot unoccupancy period. The spectrum decision structure initially discovers the spectrum slot occupancy position, unoccupancy period of the spectrum slot by using Minimum Mean Square Error (MMSE) and Blind Source Separation (BSS) strategy under ED is used in SS system and spectrum slot execution depended on its ergodic capacity as exhibited in Figure 3.

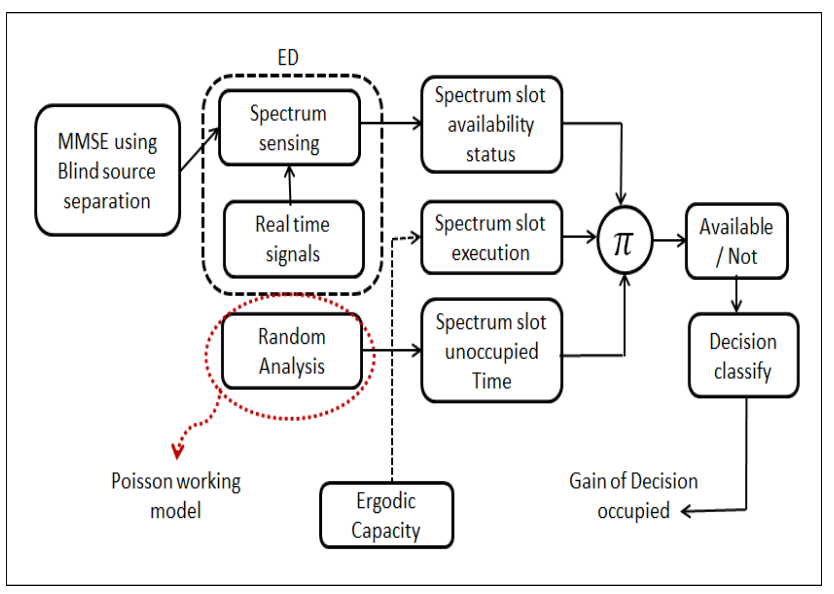

Fig. 3.Suggested spectrum decision model for CRN depended IoT in 5G/B5G networks

An optimum spectrum sensing technique is developed for the CRN based IoT in 5G/B5G networks through the MMSE along with the BSS approach. The impact caused by the channel ambiguity is reduced by an optimal MMSE detector to enhance the detection operation according to the sensible spectrum sensing environment [28]. Also a BSS approach is applied together to separate the mixed channels which is in collision, and the multiple receiving antennas are needed to discover the appearance of the PU signal, unless the necessity for a quiet period of time throughout the spectrum sensing [29]. This deed improves the data assess of the SU. Thus extremely based on IoT 5G technologies. Moreover, these methods do not need any previous knowledge regarding the PU or SU signal. The new model of spectrum decision framework eliminating the silence period, and the operation of a developed algorithms depended on BSS outperforms the traditional spectrum sensing ED.

\section{A. MMSE Detector using BSS Method in Realistic Reporting Channel:}

The local spectrum sensing determination from the $k$ SUs are debased in the reporting channel having a Rayleigh fading and AWGN. The debased decisions are passed to the MMSE detector. The desired signal is found and acquired by a weight matrix $W$ which turns the impact of the channel and the MMSE weight matrix is stated as,

$$
W_{\text {MMSE }}=\left[H^{H} H+\sigma_{z}^{2} I\right]^{-1} H^{H}
$$

Where $(.)^{H}$ is the Hermitian transpose function, $\sigma_{z}^{2}$ is the statistical data of noise, $H$ is the channel matrix and $I$ is the identity matrix.

The idea of BSS divides the received signals unless the need of preceding data. One of the techniques of BSS is Independent Component Analysis (ICA). The ICA detects the isolation that causes the divided free signal. Hence, the ICA needs the supposition that the original signals are free to every other, and, this requirement is contented in communications systems. When the number of supervisions is greater than the number of sources, it is known as (over)determined BSS; otherwise, it is defined as underdetermined BSS. The (over)determined BSS linear mixture is considered in this article and necessity to inquire from the various independent supervisions; therefore the multiple antennas are needed. Therefore, the MMSE weight matrix using BSS method is rewritten as,

$$
W_{\text {MMSE }}=\eta\left[H^{H} H+\sigma_{\Sigma}^{2} I\right]^{-1} H^{H} W
$$

Where $\eta$ is the learning rate and $W$ is the estimated demixing matrix. The solution of the MMSE detector by applying the BSS method is given as,

$$
\hat{b}_{k_{M M S E-B S S}}=W_{\text {MMSE }} y_{k}
$$$$
\widehat{b}_{k_{M M S E-B S S}}=\hat{b}_{k}+\hat{\mathrm{z}}_{\hat{k}}
$$

Where $y_{k}$ is the signal received in the system and $\hat{z}_{k}$ is the AWGN among the $k^{\text {th }}$ CBS and CR. From "(3)", the desired signal $\hat{b}_{k}$ is acquired using MMSE detection using the BSS method which is distributed to the CBS to model the last decision by applying the Majority fusion logic and the end decision is informed to every SUs. The goal of BSS is to recover the source signal $\hat{b}_{k}$ from the blended signals $y_{k}$ as,

$$
\hat{b}_{k}=W_{y_{k}}=W\left(A b_{k}\right)=(W A) b_{k}
$$

By examining the above equation, it is obvious that the condition $W A=I$ (or $W=A^{-1}$ ) is raised.

The matrix of $\mathrm{A}$ is provided as,

$$
\begin{aligned}
& A=\left[\begin{array}{l}
a^{P U} \\
a^{C R}
\end{array}\right]=\left[\begin{array}{l}
a_{1} \\
a_{2}
\end{array}\right] \\
& H=\left[\begin{array}{cc}
h_{11} & h_{12} \\
\vdots & \vdots \\
h_{k 1} & h_{k 2}
\end{array}\right]
\end{aligned}
$$

Published By:

Blue Eyes Intelligence Engineering and Sciences Publication

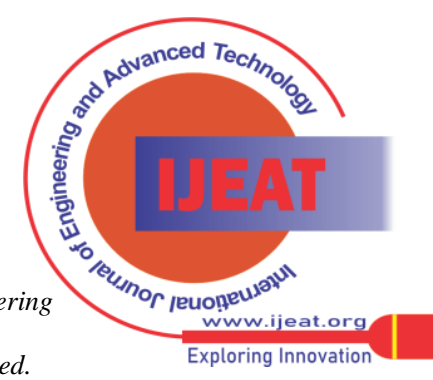


Where $a^{P N}$ is the conveyed PU symbols, $a^{C N}$ is the conveyed SU symbols. Only one of the SUs can pass the data on sensed frequency band. Then, $\mathrm{H}$ contains the channel coefficients that supposed to be constant while in the spectrum sensing and the channel coefficient among the PU and the SUs are given by, $h_{\mathrm{ii}}$ and $i$ contains both the users.

And

$$
\begin{aligned}
& a^{p N}=\left[\begin{array}{llll}
a_{1}^{P U} & a_{2}^{p U} & \ldots & a_{1}^{p U}
\end{array}\right] \\
& a^{C N}=\left[\begin{array}{llll}
a_{1}^{C N} & a_{2}^{C N} & \ldots & a_{1}^{C N}
\end{array}\right]
\end{aligned}
$$

Where $l$ is the length of the sensed symbol. Assume that only one of the SUs can send its data on sensed frequency band.

In this equation, $y_{k \mathbb{l}}$ in the $k^{\text {th }} \mathrm{SU}$ has,

$$
Y=\left[\begin{array}{llll}
y_{1,1} & y_{1,2} & \cdots & y_{1,} \\
y_{2,1} & y_{2,2} & \cdots & y_{2,1}
\end{array}\right]=\left[\begin{array}{l}
y_{1} \\
y_{2}
\end{array}\right]
$$

And $Y$ is illustrating the optimum approach with matrix A and,

$$
W=\left[\begin{array}{llll}
w_{1,1} & w_{1,2} & \ldots & w_{1, L} \\
w_{2,1} & w_{2,2} & \ldots & w_{2, L}
\end{array}\right]=\left[\begin{array}{l}
w_{1} \\
w_{2}
\end{array}\right]
$$

These evaluated nongaussianity in "(2)" is then caused similarity with the predefined threshold $(\geq 1)$, thus it can model the current decision regarding the primary signal in the nodes within the sensing period. The decision taken is then applied to the remaining nodes which are sensed.

The figure 4 and 5 reveals the impact of MMSE detector in the CR system. The MMSE using BSS method is applied in future in the CRN based IoT in 5G system.

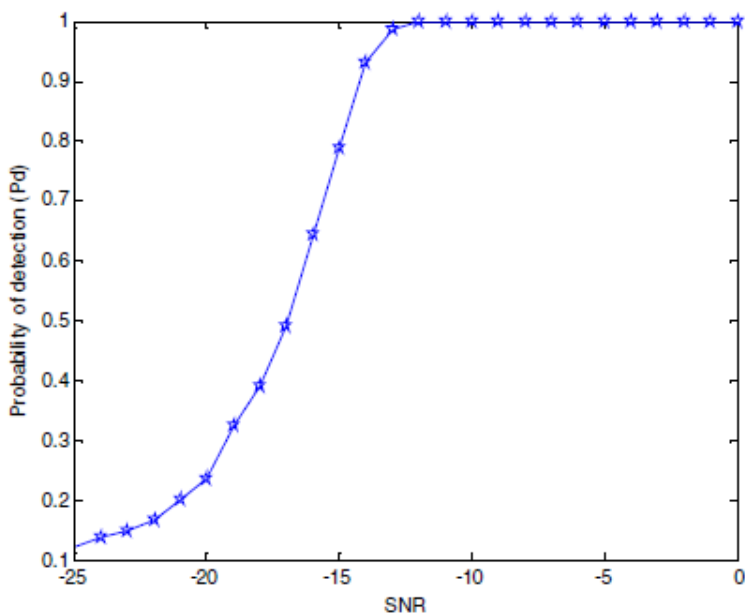

Fig. 4.Probability of detection $\left({ }^{P_{d}}\right)$ for different SNR

Fig.4 represents the probability of detection $P_{d}$ raises with the increasing SNR value [28]. This exhibits that the distance of the SUs from the PU perform an extreme crucial position in detecting the signal from the PU, so how close the SU resembles the increasing probability of detection. When think over a sensible reporting channel with flaws in the transmission mode, a MMSE detector at the CBS is applied to obtain the desired signal at the CR.

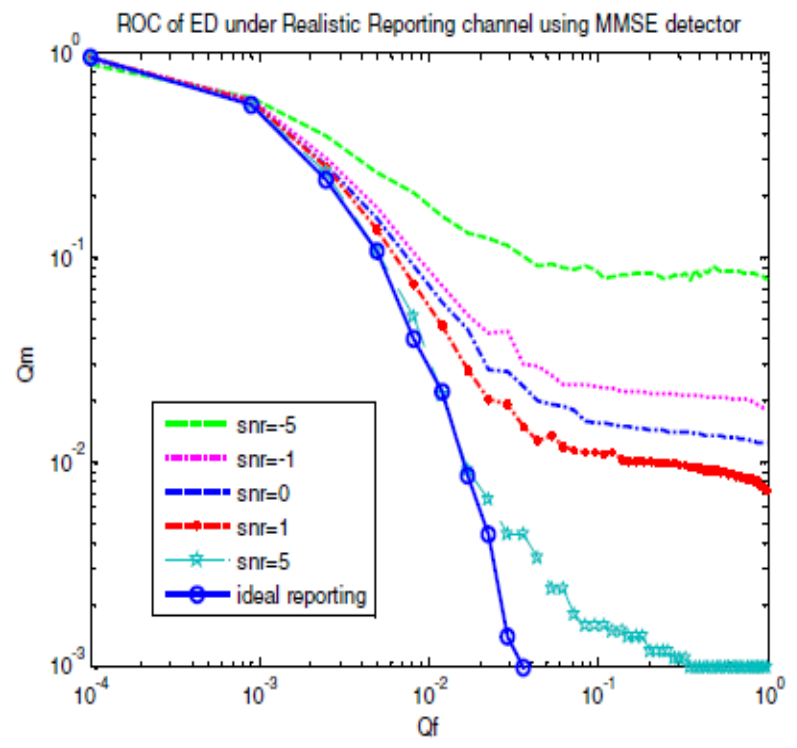

Fig. 5.Comparison of Receiver Operating Characteristic (ROC) (Probability of missed detection $\left(Q_{m}\right)$ vs Probability of false alarm) $\left(Q_{f}\right)$ for CBS functioning under noise released and sensible reporting channels having MMSE detector.

From Fig. 5, it is derived that in different SNR values, $\mathrm{SU}=5$ the execution of the MMSE detector come together with the execution of the CBS performing in a noise released surrounding with the higher SNR [28]. The MMSE optimal performance is proved in the above figures of 4 and 5 [28]. Therefore the MMSE method can be applied for the higher performance of spectrum sensing in the IoT CR based 5G/B5G networks.

\section{B. System Framework}

The Table III refers a performance of the CRN in a spectrum band ' $S$ ' having the frequency ranges from $54 \mathrm{MHz}$ to $50 \mathrm{GHz}$ that contains the majority of the wireless functions. Another description is measured such as, modulation methods, which is applied in bandwidth and transmission and an uplink - downlink frequencies [30]. The channel bandwidth is the frequency range above that an IoT-U's modem sends and receives its own signals in CRN. An IoT-U can bear its own sending's from either of the narrow, wide and UWB ranges that depends towards the wireless function and RF surroundings. The CRN contain a centralized network operator, in case a BTS that operates as 'deliver to provide' system. A section composed of 5 BTSs, an infinite number of mobile schemes and an every constructions in the surrounding are according to the review of all wireless services and these are represented in Figure 6.

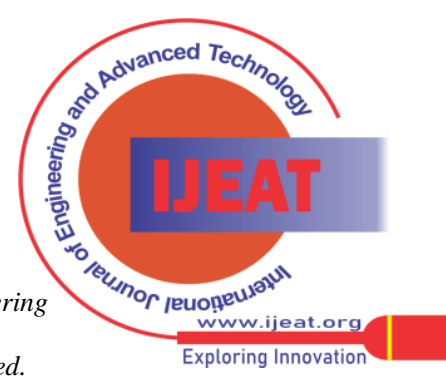




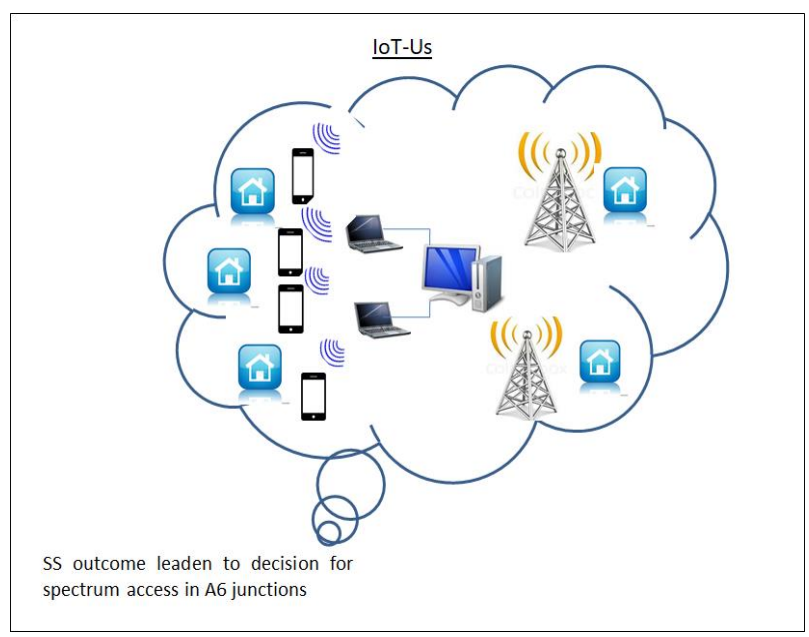

Fig. 6.System model for the spectrum decision framework

Table IV configures its radio due to the features of wireless usages for A6 junctions.

Table-IV: PU's licensed access to wireless usages

\begin{tabular}{|c|c|c|c|}
\hline Services & \multicolumn{2}{|c|}{ RF spectrum band } & Bandwidth \\
\hline \multirow[t]{2}{*}{ GSM } & \begin{tabular}{l|l} 
Uplink \\
\end{tabular} & Down link & \multirow[t]{2}{*}{$200 \mathrm{KHz}$} \\
\hline & $\begin{array}{l}890 \\
\mathrm{MHz}\end{array}$ & $95 \mathrm{MHz}$ & \\
\hline $\begin{array}{l}\text { TV Whitespace } \\
\text { (TVWS), IEEE } 802.22\end{array}$ & \multicolumn{2}{|c|}{$54 \mathrm{MHz}-862 \mathrm{MHz}$} & $6,7 \& 8 \mathrm{MHz}$ \\
\hline Zig Bee & \multicolumn{2}{|c|}{$850 \mathrm{MHz}-930 \mathrm{MHz}$} & \multirow[t]{2}{*}{ Narrowband } \\
\hline Wi-Fi, IEEE 802.11a/g & \multicolumn{2}{|c|}{$2.4 \mathrm{GHz}$} & \\
\hline UWB & \multicolumn{2}{|c|}{$3.1 \mathrm{GHz}-10.6 \mathrm{GHz}$} & $500 \mathrm{MHz}$ \\
\hline Personal & Uplink & Down link & \multirow[t]{2}{*}{$30 \mathrm{MHz}$} \\
\hline $\begin{array}{l}\text { Communications } \\
\text { Services (PCS) }\end{array}$ & $\begin{array}{l}1710-1780 \\
\mathrm{MHz}\end{array}$ & $\begin{array}{l}1805-1860 \\
\mathrm{MHz}\end{array}$ & \\
\hline US paging & \multicolumn{2}{|c|}{$931 \mathrm{MHz}-932 \mathrm{MHz}$} & \\
\hline $\begin{array}{l}\text { Global Positioning } \\
\text { System (GPS) }\end{array}$ & \multicolumn{2}{|c|}{$\begin{array}{l}\text { L1: } 1375.42 \mathrm{MHz} \\
\text { L2: } 1227.60 \mathrm{MHz}\end{array}$} & $1 \mathrm{MHz}$ \\
\hline $\begin{array}{l}\text { Industrial, scientific and } \\
\text { medical networks (ISM) }\end{array}$ & \multicolumn{2}{|c|}{$\begin{array}{l}902-928 \mathrm{MHz} \\
2.4-2.484 \mathrm{GHz} \\
5.725-5.850 \mathrm{GHz}\end{array}$} & Narrowband \\
\hline \multirow[t]{2}{*}{ CDMA } & Uplink & $\begin{array}{l}\text { Down link } \\
\end{array}$ & \multirow[t]{2}{*}{$1.25 \mathrm{MHz}$} \\
\hline & $\begin{array}{l}\text { 824-849 } \\
\text { MHz }\end{array}$ & $\begin{array}{l}\text { 869-894 } \\
\text { GHz }\end{array}$ & \\
\hline WCDMA & $\begin{array}{l}\text { 1900-1920 } \\
\mathrm{MHz}\end{array}$ & $\begin{array}{l}2020-2025 \\
\mathrm{MHz}\end{array}$ & $5 \mathrm{MHz}$ \\
\hline
\end{tabular}

\section{Experimental Results}

The experimental result for the probability of detection of different SNR using MMSE detector is shown in Table V.

Table-V: Probability of detection for different SNR using MMSE detector

\begin{tabular}{|c|c|}
\hline $\begin{array}{c}\text { SNR } \\
(\mathbf{d B})\end{array}$ & $\begin{array}{c}\text { Probability of } \\
\text { detection }\left(\boldsymbol{P}_{\boldsymbol{d}}\right)\end{array}$ \\
\hline 0 & 1 \\
\hline-5 & 1 \\
\hline-10 & 1 \\
\hline-15 & 0.8 \\
\hline-20 & 0.24 \\
\hline-25 & 0.12 \\
\hline
\end{tabular}

The experimental results for the comparison of ROC for CBS functioning under noise released and sensible reporting channels having MMSE detector is shown in Table VI.
Table-VI: Comparison of $\left(Q_{m}\right)$ and $\left(Q_{f}\right)$ for the CBS

functioning under noise released and sensible reporting channels having MMSE detector

\begin{tabular}{|c|c|c|c|c|c|c|}
\hline \multirow{3}{*}{$\begin{array}{c}\text { Probability } \\
\text { of false } \\
\text { alarm } \\
\text { detection } \\
\left(Q_{\rho}\right)\end{array}$} & \multicolumn{6}{|c|}{ Probability of missed detection $\left(Q_{m}\right)$} \\
\hline & \multirow{2}{*}{$\begin{array}{c}\text { Ideal } \\
\text { Reporting }\end{array}$} & \multicolumn{5}{|c|}{ SNR (dB) } \\
\hline & & $\begin{array}{c}S N R= \\
5\end{array}$ & $\begin{array}{c}S N R= \\
1\end{array}$ & $\begin{array}{c}S N R= \\
0\end{array}$ & $\begin{array}{l}\text { SNR } \\
=(-1)\end{array}$ & $\begin{array}{l}\text { SNR } \\
=(-5)\end{array}$ \\
\hline $10^{0}$ & $>>0.001$ & 0.001 & 0.090 & 0.020 & 0.039 & $\begin{array}{l}0.09 \\
8\end{array}$ \\
\hline $10^{-1}$ & $>>0.001$ & 0.003 & 0.019 & 0.040 & 0.045 & 0.10 \\
\hline $10^{-2}$ & 0.035 & 0.080 & 0.084 & 0.089 & 0.12 & 0.24 \\
\hline $10^{-3}$ & 0.85 & 0.86 & 0.87 & 0.88 & 0.89 & 0.91 \\
\hline $10^{-4}$ & 1 & 1 & 1 & 1 & 1 & 1 \\
\hline
\end{tabular}

\section{CONCLUSION AND FUTURE WORK}

The CR is a significant assessment for the troublesome of spectrum scarcity troublesome. To use the previously allotted spectrum, the spectrum decision includes an importance of $\mathrm{CR}$. The spectrum decision allows the CR users to access the spectrum slots due to the wireless persistence on a broader range of RF spectrum. Hence, the optimum method for spectrum decision is significant for the CR based IoT in 5G system. Therefore, the paper suggest a novel SS technique and a possible spectrum decision model that measures the spectrum band from its unoccupied time, execution and occupancy position. Also, it assured A6 junction by means of that supplying a possible technology for IoT to favour 5G/B5G networks which having larger data rates. In future, this novel SS technique and the spectrum decision model is implemented in the IoT based 5G/B5G system for the possible convenient of the network model.

\section{REFERENCES}

1. L. Atzori, A. Iera, and G. Marobito, "The internet of things: A survey," Computer Networks, 54(15), 2010, pp. 2787-2805.

2. X. Liu, D. He, and M. Jia, "5G-based wideband cognitive radio system design with cooperative spectrum sensing," Physical Communication 25(2), 2017, pp. 539-545.

3. A. Roy, S. Sengupta, K-K. Wong, V. Raychoudhury, K. Govindan, and S. Singh, "5G wireless with cognitive radio and massive IoT," IETE Technical Review, 34(suppl 1), 2017, pp. 1-3.

4. K.S. Shree, E.B. Tadilo, B.L. Long, C. Symeon, W. Xianbin, and O. Bjorn, "Dynamic spectrum sharing in $5 \mathrm{G}$ wireless networks with full duplex technology- Recent advances and research challenges," IEEE Communication Surveys and Tutorials, 20(1), 2018, pp. 674-707.

5. C.I. Badoi, N. Prasad, V. Croitoru, and P. Ramjee, " $5 \mathrm{G}$ based on cognitive radio," Wireless Personal Communications, 57(3), 2011, pp 441-464.

6. M. Agiwal, A. Roy, and N. Saxena, "Next generation 5G wireless networks: A comprehensive survey," IEEE Communication Surveys and Tutorials, 18(3), 2016, pp. 1617-1655.

7. Cisco, "Cisco Visual Networking Index: Global Mobile Data Traffic Forecast Update," Cisco Annual Internet Report, White paper (2018-2023), June 2018. https://newsroom.cisco.com/press-release-content?type=webcontent\&a rticleId=1912960

8. C. Perera, A. Zaslavsky, P. Christen, and D. Geogakopoulos, "Context aware computing for internet of things: A survey," IEEE Communication Surveys and Tutorials, 16(1), 2014, pp. 414-454.

9. Saha, B., "Fog computing - A Bridge in cloud-to-thing continuum of IoT," Mobiloitte, Internet of Things, August 2018.

Published By:

Blue Eyes Intelligence Engineering and Sciences Publication

(C) Copyright: All rights reserved.

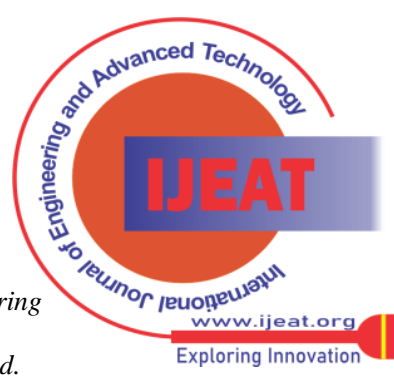


10. https://www.mobiloitte.com/blog/fog-computing-a-bridge-in-cloudtothing-continuum-of-iot/

11. A. Habibzadeh, S. Shirvani Moghaddam, S.M. Razavizadeh, and M. Shirvanimoghaddam, "Modeling and analysis of traffic-aware Spectrum handover schemes in cognitive Het nets," Transactions on Emerging Telecommunications Technologies (ETT), 28(12), 2017, pp. 1-3.

12. A.A. Khan, M.H. Rehmani, and A. Rachedi, "When cognitive radio meets the internet of things?," In Proc. International Wireless Communications and Mobile Computing Conference, Paphos, 2016, pp. 469-474.

13. S. Sabat, P.K. Sharma, and A. Gandhi, "Full duplex cooperative Spectrum sensing with primary user activity in cognitive radio network," IETE Technical Review, 34(sup 1), 2017, pp. 4-14.

14. M. El Tanab, and W. Hamouda, "Resource allocation for underlay cognitive radio networks: A survey," IEEE Communication Surveys and Tutorials, 19(2), 2017, pp. 1249-1276

15. W. Y. Lee, and I. Akyildiz, "A spectrum decision framework for cognitive radio networks," IEEE Transactions on Mobile Computing, 10(2), 2011, pp. 161-174.

16. W. Y. Lee, and I. F. Akyildiz, "Spectrum-aware mobility management in cognitive radio cellular networks," IEEE Transactions in Mobile Computing, 11(4), 2012, pp. 529-542.

17. A. N. Akhtar, A. Rashdi, and F. Arif, "Fusion based spectrum decision framework for cognitive radio users," Proc., International Symposium on A World of Wireless, Mobile and Multimedia Networks, Boston, MA, 2015, pp. 1-6.

18. D. Bohning, Notice of Proposed Rule Making and Order. FCC, ET Docket no. 03-222, September 2003, pp. 197-200. https://www.fcc.gov/general/rulemaking-fcc

19. M. T. Masonta, M. Mzyece, and N. Ntlatlapa, "Spectrum decision in cognitive radio networks: A survey," IEEE Communication Surveys and Tutorials, 15(3), 2013, pp. 1088-1107.

20. L. Le, and E. Hossain, "Resource allocation for spectrum underlay in cognitive radio network," IEEE Transactions on Wireless Communications, 7(12), December 2008, pp. 5306-5315.

21. Z. Junni, X. Hongkai, W. Dawei, and C. Chang-Wen, "Optimal power allocation for hybrid overlay/underlay spectrum sharing in multiband cognitive radio network," IEEE Transactions on Vehicular Technology, 62(4), 2013, pp. 1827-1837.

22. S. Haykin, "Cognitive radio: Brain-empowered wireless communications," IEEE J. on Selected Areas in Communications, 23(2), 2005, pp. 201-220.

23. L. Lu, X. W. Zhou, U. Onunkwo, and G. Y. Li, "Ten years of cognitive radio technology," EURASIP Int. J. of Wireless Communication Networking, 28(1), 2012, pp. 1-16.

24. S. Shirvani Moghaddam, and M. A. Kamarzarin, "Comparative study on the two popular cognitive radio Spectrum sensing methods: Matched filter versus energy detector," American J. of Mobile Systems, Applications, and Services, 1(2), October 2015, pp. 132-139. http://www.aiscience.org/journal/ajmsas

25. A. Singh, M. R. Bhatnagar, and R. K. Mallik, "Performance of an improved energy Detector in multihop cognitive radio network," IEEE Transactions on Vehicular Technology, 65(2), 2016, pp. 732-743.

26. W. L. Chin, J. M. Li, and H. H. Chen, "Low-complexity energy detection for spectrum sensing with random arrivals of primary users," IEEE Transactions on Vehicular Technology, 65(2), February 2016, pp. 947-952.

27. H. Sun, A. Nallanathan, C. X. Wang, and Y. Chen, "Wideband spectrum sensing for cognitive radio networks: A survey," IEEE Wireless Communications, 20(2), April 2013, pp. 74-81.

28. A. N. Akhtar, A. Ghafoor, A. Rashdi, and F. Arif, "Fuzzification supported spectrum decision framework for cognitive radio networks,' Transactions on Emerging Telecommunications, 28(4):e3229, 2017, pp $1-3$.

29. J. Liza, K. Muthumeenakshi, and S. Radha, "Cooperative Spectrum Sensing in a Realistic Cognitive Radio Environment," proc. International Conference on Recent Trends in Information Technology, MIT, Anna University, Chennai, June 2011, pp. 375-379.

30. A. Nasser, "Blind Source Separation-based Full-Duplex Cognitive Radio," Int. J. of Digital Information and Wireless Communications, 8(1), 2018, pp. 47-51

31. Theodore S. Rappaport, Wireless communications: principles and practice (2nd ed). Prentice Hall PTR, Cloth, Upper Saddle River, NJ, Great Britain, 2002.

\section{AUTHORS PROFILE}

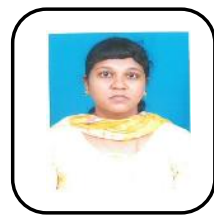

A. Hyils Sharon Magdalene, is an Assistant Professor in the department of Computer science \& Engineering in PSN College of Engineering \& Technology, Tirunelveli. She received her Bachelor and Master degrees in the Faculty of Information and Communication Technology at Manonmaniam Sundaranar University, Tirunelveli. She has presented national and international conferences and has published many national and International journals. Her research interest includes, network security, wireless network and computer communication.

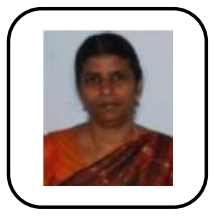

Dr. D. Shalini Punithavathani, received her Bachelor of Science, in 1979 from Sarah Tucker College, affiliated to Madurai Kamarajar University, India. She obtained her Bachelor of Technology in Electronics, in 1982 from Madras Institute of Technology, Chennai affiliated to Anna University, Chennai, India. And further the Master of Engineering in Computer Science and Engineering, in 1990 from Government College of Technology, Coimbatore affiliated to Bharathiyar University, Coimbatore, India. She was awarded with Doctorate in Philosophy, entitled "Study and Implementation of IPv4 to v6 translation techniques" in 2010 in Anna University, Chennai, India. She has present several conferences and published several Journals. Currently, she is a retired principal of Government College of Engineering, Tirunelveli, Tamil Nadu. Her professional areas of interests are mobile computing, Networking and Network security.

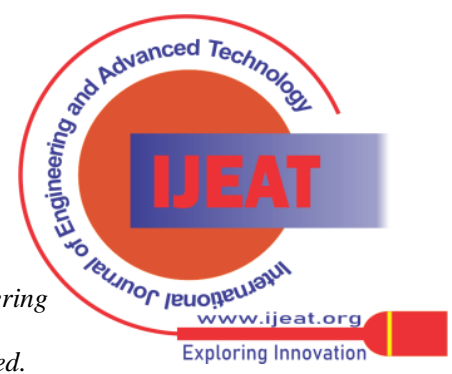

